\title{
Are we teaching our hospitalized patients self-management skills? Opportunities lost
}

\author{
Serena Miotto ${ }^{1,2}$, Lynn McNicoll2,3,4, Kristen Butterfield ${ }^{2,3}$, Christina Rincon ${ }^{2,3}$, Mriganka Singh M, $^{3}$, Stefan \\ Gravenstein*2, 3,4,5 \\ ${ }^{1}$ University of Padua, Padua, Italy \\ ${ }^{2}$ Healthcentric Advisors, Providence, Rhode Island, United States \\ ${ }^{3}$ Rhode Island Hospital, Department of Medicine, Providence, Rhode Island, United States \\ ${ }^{4}$ Warren Alpert Medical School of Brown University, Providence, Rhode Island, United States \\ ${ }^{5}$ University Hospitals-Case Medical Center and Case Western Reserve University, Cleveland, Ohio, United States
}

Received: November 8, 2014

DOI: $10.5430 /$ jha.v4n2p30
Accepted: December 22, 2014 Online Published: January 30, 2015

URL: http://dx.doi.org/10.5430/jha.v4n2p30

\begin{abstract}
Background: In the U.S., $20 \%$ of medical hospital discharges are readmitted within 30 days. Non-medical intervention strategies, such as teaching four specific self-management skills, have demonstrated reduction in readmission risk by a third or more. Objective: To investigate whether hospitalized patients are taught or learn four basic self-management skills ("four skills") during routine clinical encounters.

Methods: Design: Observational study from May to October 2012 in an academic teaching hospital. Participants: Consenting medical patients (aged 18 or over), their visitors or caregivers and their providers. Unit managers identified patients who would be discharged home and capable of learning the four skills. Interventions: A trained observer consented patients and monitored every provider encounter for mentioning and/or teaching of the four specific self-management skills' content for intervals of up to 6 hours during daytime shifts. After each encounter, the observer queried the patient and their caregiver to see what information relating to the four skills they retained and understood. Additionally, observers recorded patient demographics, native language, provider type, and major medical conditions present at the time of the encounter. Five control activities expected to occur routinely for each encounter were monitored for comparison. Main measures: Frequency of one of four self-management skill education events during patient-provider encounters.

Results: We observed 56 patients over 326 encounters involving 117 physicians or medical students, 134 nurses or nursing students, and 163 hospital staff. Among 189 encounters with clinical staff (physicians or nurses), the four basic self-management skills were mentioned in 54 encounters $(28.6 \%)$ but taught only in 12 encounters $(6.3 \%)$. The comparison with control behaviors show a much higher proportion (hand washing 35\%-41.3\%, identified role $29.5 \%$, asking for more question $19.1 \%$ ). Physicians are more likely than nurses to discuss clinical conditions, medications, or any of the four skills' topics. Specific self-management teaching of patients led to better understanding of the these skills compared to just mentioning them $(p=.03)$.

Conclusions: Hospital providers rarely teach four basic self-management skills as part of regular care. Patients can learn this content while hospitalized which could potentially reduce 30-day readmission risk.
\end{abstract}

Key Words: Patient education, Rehospitalizations, Quality improvement

*Correspondence: Stefan Gravenstein; Email: Stefan.Gravenstein@uhhospitals.org; Address: Mailstop HAN 6095, 11100 Euclid Ave, Cleveland, $\mathrm{OH}$, United States. 


\section{Introduction}

In the United States, depending on the clinical condition and geographic region, about $20 \%$ of fee-for-service Medicare patients are readmitted within 30 days of hospital discharge. ${ }^{[1,2]}$ Over one third of these readmissions come from home and may be avoidable by better self-management preparation. ${ }^{[1,3-5]}$ This is also true with specific conditions, such as heart failure, that require more diligent selfmanagement skill to stave off rehospitalization. In one example, a 1-hour teaching session program before hospital discharge was associated with a $35 \%$ lower readmission and death outcome. ${ }^{[6]}$ Another study based on a patient education and support intervention without medical management components reported similar results. ${ }^{[4]}$

In 2006, Coleman et al., reported specific education of patients discharged home reduced readmission risk by a third. ${ }^{[3]}$ The replication of this approach in an open health care system, demonstrating the real-world application of this approach, again reduced readmission risk by over $30 \% .^{[1]}$ The approach, coined as the Care Transitions Intervention (CTI), is anchored on in-home education that takes about an hour, and three follow up phone calls. The CTI involves no medical intervention but focuses on empowering high-risk patients toward better illness self-management. Remarkably, most of the other promising approaches to reduce readmissions do much more with no better outcome. ${ }^{[5,7]}$ Coleman's CTI centrally involves coaching self-management content through four basic selfmanagement skills:

(1) patient's medical problem list;

(2) medications;

(3) warning signs of impending medical crisis ("red flags");

(4) how to get timely access to care recorded by the patient or caregiver.

Currently, hospital staff prepare after-hospital care plans that they review with the patient or their caregivers to ensure a safe discharge home. Thus, at the time of hospital discharge, hospital staff must believe their patients are adequately prepared to self-manage until the next planned outpatient clinical encounter, or they would or should not release their patient to go home. The success of the CTI and similar educational interventions suggests there is room for improvement. Since basic post-hospital education seems to reduce rehospitalization, we hypothesized that basic selfmanagement understanding often remains poor at the time of discharge. We cannot tell whether poor understanding of CTI-like content is due to a lack of teaching the material to the patient or lack learning taught CTI content on the part of the patient. More specifically, we aimed to evaluate whether (1) hospital providers teach any content of these four basic self management skills ("four skills") as part of regular care to patients and their caregivers, and (2) whether information presented regarding any of the four skills' is understood and retained after the provider leaves the room. Secondarily, the project gathers information about standard or "control" care behaviors, such as hand washing on room entry or exist, ${ }^{[8]}$ and quality of the provider-patient interaction ${ }^{[9]}$ to capture a baseline rate for five "good clinical practice" activities.

\section{Methods}

\subsection{Study population}

We performed this study at an academic teaching hospital from May to October 2012. The Lifespan Institutional Review Board approved the study. We observed three types of consenting subjects: (1) hospitalized medical patients over the age of 18 ; (2) the visitors and informal caregivers present during hospital care encounters with the patient; and (3) any healthcare professional, including physicians, nurses or hospital staff providing face-to-face care to these patients. Henceforth, we refer to each of these categories of subjects as patients, visitors and providers. The study excluded patients not capable of giving consent and those patients living in long-term care settings.

\subsection{Study design}

This observational study assessed patient-provider interaction in the patient's hospital room. An observer counted how often any provider mentioned any content of one of four specific self-management skills ("four skills") at each encounter. We defined an "encounter" as any direct face-toface interaction between the patient and one or more health professionals (such as physicians, nurses, dieticians, etc.). If multiple providers were present concurrently during any part of an encounter, we considered it a single encounter.

\subsection{Data collection tools}

We constructed the data collection tool, a case report form, to tally details of observations on the web-based survey tool, SurveyMonkey ${ }^{\circledR}$ (Palo Alto, CA). For every providerpatient encounter, the observer completed one observation report; thus multiple report forms were typically completed for each patient over the course of up to a 6-hour observation period. The case report form also recorded a patient ID number linked to the observation date, time and observer; thus, it allowed the linkage of multiple encounters for a specific patient. The form displayed the four basic self-management skills separately for cross-reference by provider at the encounter.

\subsection{Observation procedure}

Observers were either physicians, non-clinical hospital employees or volunteers. All were trained by one of the two authors (SM and SG) to consent patients and observe patientprovider encounters. Training consisted of a teaching phase 
and a practical one; in the practical phase, the trainer and the trainee performed two paired observations of the same patients, then compared results to minimize the observation bias. In our study, a total of three observers were involved.

Daily, observers selected two hospitalized patients on their unit from a list the nurse hospital unit manager provided who met the inclusion criteria for our study. If the first patient approached declined consent, we approached the other. The observer hung a flyer on the consented patient's hospital room describing the goal of the study and the presence of an observer to anyone entering the room. The observer wore a clearly visible nametag to identify themselves and their role to the patient and to anyone entering the room.

During the observation period, the observer recorded if a provider presented the content of one (or more) of the four basic self-management skills. The observer subjectively differentiated if the content was taught to the patient or simply mentioned, depending on the extent of the explanation and details presented. Following the encounter, the observer asked the patient and visitor/caregiver, if present, what they could recall about any of the four skills taught to determine what knowledge they acquired.

For control behaviors, we considered activities expected to occur routinely or always for each encounter ("always events"): providers identifying themselves and their role during a patient care encounter, hand washing on room entering and exit, immediate care plan for the day reviewed, laboratory testing plans reviewed, and offering the patients or their visitors the opportunity to ask additional questions.

During the observation period, the observer recorded: (1) patient demographics (patient's room number, sex, age group), (2) native language (and if an interpreter was needed). As the observer witnessed an encounter, the observer populated an encounter form and recorded: (1) encounter initiation and end time, (2) visitor information if a visitor was present, (3) provider present at the encounter, (4) whether the provider taught or mentioned any element of the four self-management skills, (5) major medical conditions present at the time of the encounter as elicited at the bedside, and (6) control behaviors.

The observation period lasted no more than a single, sixhour period for any patient participant. Observers staggered their schedules during daytime hours and across several wards, to observe a wide variety of provider-patient interactions.

\subsection{Consent process}

Among the three studied subject categories (patients, visitors and providers), only patients gave consent both orally and in writing. Verbal consent was obtained for the others, who were given the opportunity to ask the observer to leave the room for their patient encounter, i.e., to decline to con- sent. Providers received e-mail notification in advance of the study supporting its importance by hospital and physician staff leadership, and were asked to allow observation of their bedside encounters. A copy of the data collection tool was included in this e-mail. Also, fliers announcing the study were placed in staff work areas ahead of the start of the study. Asking the observer to step out during the encounter was taken as declination by the provider to participate in the study.

\subsection{Statistical analysis}

We grouped providers into three categories. The first category was "physicians" and included attending physicians, consultants, residents and medical students. The second category was "nurses" and included registered nurses, licensed practice nurses and nursing students. The third category was "hospital staff" and included certified nursing assistants, pharmacists, dieticians, housekeepers, transport personnel and others. Some encounters had different provider types present at once; in this case we considered it a physician encounter if a physician was present, and a nurse encounter if a physician was not present.

We analyzed data at the patient level for characteristics related to patients $(\mathrm{N}=56)$, and also at the encounter level, for characteristics related to the specific encounter $(\mathrm{N}=$ 326). Frequencies and counts were reported for patient and provider characteristics. We used chi-square tests for categorical variables to determine the association between behaviors and provider characteristics, and Student's t-tests for continuous variables. All data were analyzed using SAS version 9.2 (Cary, NC).

\section{Results}

\subsection{Patient demographics}

We observed 56 patients, $55 \%$ male and $45 \%$ female. The prevalent age group was 51-65 years old (39\%) and $44.7 \%$ of our cohort was over 65 years of age. Most of our patients spoke English (86\%); only eight patients had English as a second language and half of these could communicate without an interpreter present. The top eight conditions discussed between patients and providers were: pain $(39.3 \%)$, cardiac problems $(17.9 \%)$, gastrointestinal problems $(14.3 \%)$, diabetes $(12.5 \%)$, infections (12.5\%), pulmonary problems $(8.9 \%)$, diarrhea $(7.1 \%)$ and blood pressure abnormality $(5.4 \%)$ (see Table 1$)$.

\subsection{Encounter characteristics}

We observed 326 encounters between patients and the hospital staff. There were 79 encounters with doctors $(24 \%)$, 110 with nurses (34\%) and 137 with hospital staff (42\%). Most of our observations occurred on a physician teaching service $(80 \%)$ in a room without isolation precautions 
$(89 \%)$. We observed visitors during only a few encounters (10\%): seven of these included a primary caregiver and 23 a family member. Most of our observations occurred on Tuesdays and Fridays and between 10:00 am and 1:00 pm (see Table 2).

Table 1: Patient characteristics $(\mathrm{N}=56)$

\begin{tabular}{lll}
\hline Characteristics & N & Percent \\
\hline Gender & 31 & 55.4 \\
Male & 25 & 44.6 \\
Female & & \\
Age & 9 & 16.1 \\
$18-50$ & 22 & 39.3 \\
$51-65$ & 9 & 16.1 \\
66-75 & 16 & 28.6 \\
$76+$ & & \\
Language & 48 & 85.7 \\
English as First & 8 & 14.3 \\
English as Second & & \\
If English Second & 4 & 50.0 \\
Patient Needs Interpreter & 4 & 50.0 \\
No Interpreter Needed & & \\
Major Medical Condition & 22 & 39.3 \\
Pain & 10 & 17.9 \\
Cardiac problem & 8 & 14.3 \\
Gastroenterologic (no bleed) & 7 & 12.5 \\
Diabetes & 7 & 12.5 \\
Infections & 5 & 8.9 \\
Pulmonary (not asthma, pneumonia) & 4.1 \\
Diarrhea & 3 & 5.4 \\
Blood pressure abnormality & 39.3 \\
Pain & 22 & \\
\hline & & \\
\hline
\end{tabular}

\subsection{Always events}

Among the 79 encounters with physicians, we observed a total of 117 physicians. Among the 110 encounters with nurses, we observed 134 nurses. Among the 137 observations with hospital staff, we observed 163 hospital staff. We counted the frequency of control behaviors (always events) according to provider type. Results show that physicians are significantly more likely than nurses to identify their role (31.6\% vs. $21.6 \%, p<.05)$, wash hands on entering $(52.1 \%$ vs. $38.8 \%, p<.05)$ and exiting $(54.7 \%$ vs. $55.2 \%, p<$ $.05)$ the room, ask the patient if they had any more questions $(44.4 \%$ vs. $11.9 \%, p<.05)$, review the plan for the day $(25.6 \%$ vs. $5.2 \%, p<.05)$ and discuss lab tests $(27.4 \%$ vs. $4.5 \%, p<.05)$. Results show physicians are also significantly more likely than hospital staff to wash hands on entering $(52.1 \%$ vs. $19.6 \%, p<.05)$ and exiting the room (54.7\% vs. $20.2 \%, p<.05$ ), ask the patients if they had any more questions $(44.4 \%$ vs. $6.7 \%, p<.05)$ review the plan for the day $(25.6 \% v s .1 .8 \%, p<.05)$ and discuss lab tests $(27.4 \%$ vs. $0.6 \%, p<.05)$. Nurses are significantly more likely than hospital staff to identify their role $(21.6 \%$ vs. $34.4 \%, p<.05)$, wash hands on entering $(38.8 \%$ vs. $19.6 \%, p<.05)$ and exiting $(55.2 \%$ vs. $20.2 \%, p<.05)$ the room, and discuss lab tests $(4.5 \%$ vs. $0.6 \%, p<.05)$.

Table 2: Encounter characteristics $(\mathrm{N}=326)$

\begin{tabular}{lll}
\hline Characteristics & N & Percent \\
\hline Service & & \\
Teaching & 260 & 79.8 \\
Isolation Precautions Posted & & \\
Yes & 35 & 10.7 \\
Provider Type Present & & \\
MD Attending & 39 & 12.0 \\
MD Resident & 45 & 13.8 \\
MD Student & 18 & 5.5 \\
MD Consultant & 15 & 4.6 \\
RN/LPN/Nursing student & 134 & 41.1 \\
Other & 163 & 50.0 \\
Day of Week & & \\
Monday & 14 & 4.3 \\
Tuesday & 136 & 41.7 \\
Wednesday & 50 & 15.3 \\
Thursday & 49 & 15.0 \\
Friday & 77 & 23.6 \\
Time of Day & & \\
Morning (8am-1pm) & & \\
Afternoon (1pm-6pm) & 244 & 74.8 \\
Visitor Present At Encounter & 82 & 25.2 \\
Yes & & \\
If present, what type (n = 30) & 30 & 9.2 \\
Caregiver Only & & \\
Family Member Only & 4 & 13.3 \\
Time Spent at Encounter (mean, SD) & & \\
Physicians & 7.76 & 9.91 \\
Nurses & 10.17 & 20.03 \\
Hospital Staff & & 10.36 \\
All Providers & & \\
\hline & & \\
\hline
\end{tabular}

\subsection{Four basic self-management skills ("Four skills")}

Among 189 encounters with clinicians (see Table 3), the four basic self-management skills were mentioned in 54 encounters $(28.6 \%)$ but teaching patients about any of these four skills occurred in only 12 encounters $(6.3 \%)$. Teaching involved mainly discussion of health problems (first skill, $3.7 \%$ ) and medications (second skill, 5.3\%) while red flags and red flag urgency skills are lacking $(1.1 \%$ and 0 respectively).

When we assessed patients' understanding, results show patients were 3.5 times more likely to understand any of the 
four skills if taught rather than when the topic was just mentioned $(41.7 \%$ vs. $11.8 \%, p=.03)$.

Physicians are significantly more likely than nurses to mention conditions $(45.6 \%$ vs. $6.4 \%, p<.01)$, medication name (26.6\% vs. $9.1 \%, p=.01)$ or any of the "four skills" $(48.1 \%$ vs. $14.5 \%, p<.01)$. Physicians are significantly more likely than nurses to teach any of the four skills (11.4\% vs. $2.7 \%$, $p=.02)$ (see Figure 1).

Bivariate analysis shows no significant difference between teaching any of the four skills and teaching unit, isolation precautions posted and caregiver status. Teaching was more likely to happen in the morning compared to the afternoon (5.9\% vs. $0.7 \%, p=.01)$.

Table 3: Frequency of encounters with clinicians where basic self-management skills were mentioned taught and understood by the patient $(\mathrm{N}=189)$

\begin{tabular}{llll}
\hline Skill Topic & Skill Mentioned & Skill Taught & Skill Understood \\
\hline Skill 1: Conditions, n (\%) & $43\left(22.8^{*}\right)$ & $7(3.7)$ & $8(18.6)$ \\
Skill 2: Medications, n (\%) & $38\left(20.1^{*}\right)$ & $7(3.7)$ & $5(13.2)$ \\
Medication Name, n (\%) & $31\left(16.4^{*}\right)$ & $4(2.1)$ & $5(16.1)$ \\
Medication schedule, n (\%) & $11(5.8)$ & $3(1.6)$ & $2(18.2)$ \\
Medication Route, n (\%) & $16(8.5)$ & $3(1.6)$ & $2(12.5)$ \\
Skill 3: Red Flags, n (\%) & $4(2.1)$ & $2(1.1)$ & $1(25.0)$ \\
Skill 4: Red Flag Urgency, n (\%) & $2(1.1)$ & $0(0)$ & $0(0)$ \\
Any Skill, n (\%) & $54\left(28.6^{*}\right)$ & $12\left(6.3^{*}\right)$ & $11(19.0)$ \\
\hline
\end{tabular}

Note. ${ }^{*}$ statistically significant at $p<.05$ for physicians compared to nurses

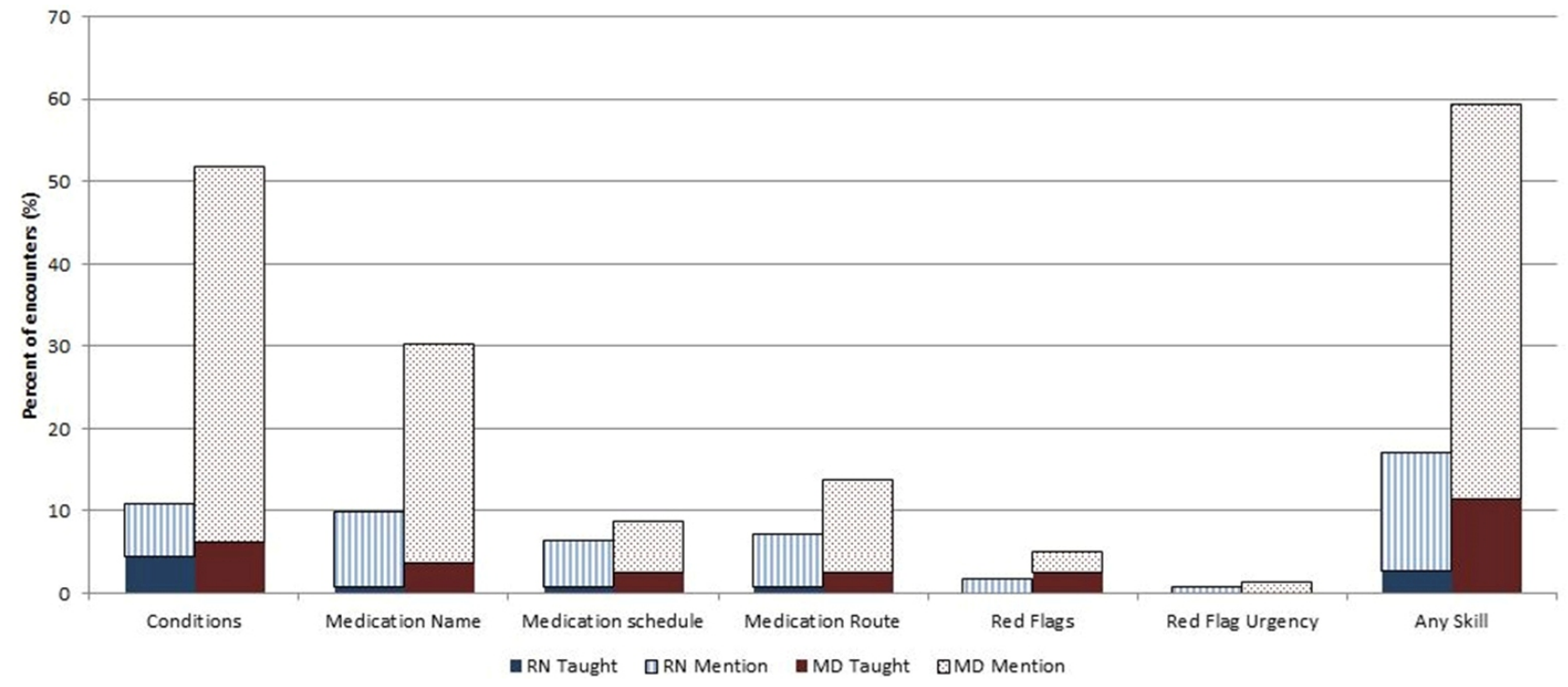

Figure 1: Percent of encounters where one of the four basic self-management skills were taught to the patient or mentioned to the patients, by providers type $(\mathrm{N}=189)$

\section{Discussion}

Our hospital providers rarely mentioned or taught any of four basic self-management skills as part of regular care. In fact, in 189 clinical encounters, teaching any of the four skills at any time occurred only 12 times. We also informally observed that many patients or their caregivers are disengaged at the time discharge instructions are offered by their providers. They are anxious to leave and not keep their ride waiting or are overwhelmed by the amount of information they need to attend to ("drinking from the fire hose"), so teaching at the time of discharge, even if it occurs, may be ineffective. In either case, the effectiveness of Coleman's CTI supports our observations that patients often leave the hospital inadequately prepared for self-management. We add to the evidence of a missed opportunity for discharge education incorporated into routine clinical encounters.

It is noteworthy that teaching basic self-management skills formally, whether while an inpatient ${ }^{[5]}$ or just after discharge as an outpatient, ${ }^{[10]}$ reduces hospital readmissions, implying effective learning occurred in both settings. Similarly, we observed informal teaching four basic skills appears more effective than just addressing them: patients' 
understanding is 3.5 times higher if these skills are taught rather than just mentioned.

Several barriers to inpatient teaching exist in the hospital environment, such as the time it takes to teach, the perception of what constitutes effective teaching, and the ownership of the teaching/learning activity. Formally teaching the four basic self-management skills we adopted from Coleman's CTI requires approximately an hour of dedicated time, assuming the learner has time to ask questions. The use of the discharge instruction form offers both structure and completeness of the information for review at discharge. The problem list, medications and follow-up care receive special attention, but teaching about their condition(s), their significance, and what medication side effects to prevent or control, does not systematically occur. It is the authors' collective experience that whatever is taught at discharge is often done with urgency, in order to complete the discharge process as quickly as possible. We propose that providers may find ways to spread the education across entire hospital stays, touching on at least one basic self-management skill at every encounter; this approach could reduce the burden of too much information at once for the patient and too much time at once for the provider while enhancing the quality of patient engagement for the entire hospital stay. For example, if nurses teach about medications when dispensing them, this could prepare the patient to discuss medication questions with the provider prior to discharge, and has the potential to identify patients in advance of discharge of a need for extra instruction.

Providers may overestimate their patients' understanding. For instance, they may assume that by mentioning a condition once, patients will remember. But, just as patients often cannot remember their provider's name or role, ${ }^{[11]}$ we show they also often will not remember mention of a condition. We conjecture that patients may be too embarrassed to ask about something they've forgotten, so the provider may never know they lack the knowledge they've attributed to them. Also, providers may assume their patient cannot understand when they may be able to. Our study suggests that when nurses identify patients who are competent to consent, these same patients are capable of learning basic selfmanagement skills if providers spend the time to explain them.

We contend that incorporating education useful for selfmanagement after discharge should begin at admission and be incorporated into routine inpatient care, like our control events. Our research project gathered information about five standard care behaviors to have a baseline rate for achievable good clinical practice. We consider these "always events" - measureable actions expected at every encounter but their occurrence varied widely. Hand washing occurred most frequently, up to half the time, and offers a potential goal for incorporating basic self management education into routine encounters, up from the $6.3 \%$ observed with clinicians. Just as hand hygiene is recognized as the key to prevent infections in the hospital, teaching the patient four basic self management skills could become part of the regular care to prevent rehospitalizations, and worthy of formal study.

\section{Limitations for the study}

Our study has several limitations. We may have overestimated provider behavior, as observed providers were aware of our research study and may have attempted to model those behaviors that might score better. We may have underestimated behaviors if teaching activities occurred outside of the observer's usual observation hours of 8:00 am to 6:00 pm, Monday to Friday; teaching likely occurs during prerounding or during medication passes which may have been missed. We may have introduced an observation bias: although observers were trained prior to the study, we cannot exclude inter-rater variability between them about observations of patient-providers encounters. Also, such patients may have been particularly in need of such education, but overwhelmed by yet another stranger (the observer), skewing our data. Finally, our observations come from a single institution, and bias cannot be mitigated from our anecdotal observations of similar approaches across several other institutions both here and abroad, making generalizability uncertain.

We have demonstrated that four basic self-management skill content is not systematically taught as part of hospital care. We have an opportunity to replicate our study to see if it indeed is as common as we suspect. We also need to determine whether inpatient the four basic management skills will translate into improving outcomes (e.g., reducing rehospitalization). We cannot establish how much we can teach patients, their level of saturation, and how much they can retain of what providers teach and explain. The important first step is to improving incorporating the four skills into routine daily encounters but further study is needed to see the impact of this on patients' behaviours or rehospitalization rate.

\section{Conflicts of Interest Disclosure}

The authors declare they have no conflicts of interest in the research, including relevant financial interests, activities, relationships, or affiliations. 


\section{References}

[1] Voss R, Gardner R, Baier R, Butterfield K, Lehrman S, Gravenstein $\mathrm{S}$. The care transitions intervention: translating from efficacy to effectiveness. Arch Int Med. 2011; 171(14): 1232-1237. PMid: 21788540. http://dx.doi.org/10.1001/archinternm ed. 2011.278

[2] Jencks SF, Williams MV, Coleman EA. Rehospitalizations among patients in the Medicare fee-for-service program. N Engl J Med. 2009; 360(14): 1418-28. PMid: 19339721. http://dx.doi.org /10.1056/NEJMsa0803563

[3] Coleman EA, Parry C, Chalmers S, Min SJ. The care transitions intervention: results of a randomized controlled trial. Arch Intern Med. 2006; 166(17): 1822-8. PMid: 17000937. http://dx.doi .org/10.1001/archinte.166.17.1822

[4] Krumholz HM, Amatruda J, Smith GL, Mattera JA, Roumanis SA, Radford MJ, et al. Randomized trial of an education and support intervention to prevent readmission of patients with heart failure. J Am Coll Cardiol. 2002 Jan 2; 39(1): 83-9. http://dx . doi .org/1 $0.1016 / \mathrm{S} 0735-1097$ (01) 01699-0

[5] Jack BW, Chetty VK, Greenwald JL, Sanchez GM, Johnson AE. A reengineered hospital discharge program to decrease rehospitalization: a randomized trial. Ann Intern Med. 2009 Feb 3; 150(3): 17887. PMid: 19189907. http://dx.doi.org/10.7326/0003-481 9-150-3-200902030-00007

[6] Koelling TM, Johnson ML, Cody RJ, Aaronson KD. Discharge education improves clinical outcomes in patients with chronic heart failure. Circulation. 2005 Jan 18; 111(2): 179-85. Epub 2005 Jan 10. PMid: 15642765. http://dx.doi.org/10.1161/01.CIR.0000 151811.53450.B8

[7] Naylor MD, Brooten DA, Campbell RL, Maislin G, McCauley KM, Schwartz JS. Transitional care of older adults hospitalized with heart failure: a randomized, controlled trial. J Am Geriatr Soc. May 2004; 52(5): 675-84. PMid: 15086645. http://dx.doi.org/10.1111 $/ j .1532-5415.2004 .52202 . x$

[8] Chemaly RF, Simmons S, Dale C Jr, Ghantoji SS, Rodriguez M, Gubb J, et al. The role of the healthcare environment in the spread of multidrug-resistant organisms: update on current best practices for containment. Ther Adv Infect Dis. 2014 Jun; 2(3-4): 79-90. PMid: 25469234.

[9] Kevin J. O'Leary, MD, MS, Nita Kulkarni, MD, Matthew P. Landler, MD, Jiyeon Jeon, MPH, Katherine J. Hahn, BS, Katherine M. Englert, et al. Hospitalized Patients' Understanding of Their Plan of Care. Mayo Clin Proc. Jan 2010; 85(1): 47-52. PMid: 20042561. http://dx.doi.org/10.4065/mcp. 2009.0232

[10] Coleman EA, Smith JD, Frank JC, Min SJ, Parry C, Kramer AM. Preparing patients and caregivers to participate in care delivered across settings: the Care Transitions Intervention. J Am Geriatr Soc. 2004 Nov; 52(11): 1817-25. PMid: 15507057. http://dx.doi.o $\mathrm{rg} / 10.1111 / \mathrm{j} .1532-5415.2004 .52504 . \mathrm{x}$

[11] Arora V, Gangireddy S, Mehrotra A, Ginde R, Tormey M, Meltzer D. Ability of hospitalized patients to identify their in-hospital physicians. Arch Intern Med. 2009 Jan 26; 169(2): 199-201. PMid: 19171817. http://dx.doi.org/10.1001/archinternm ed. 2008.565 